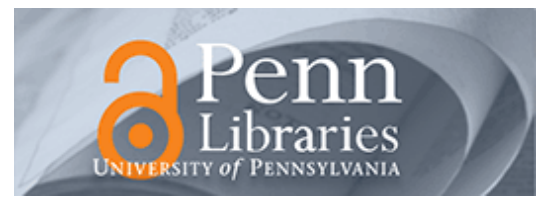

University of Pennsylvania

ScholarlyCommons

$1-1-2004$

\title{
A Metamaterial Surface for Compact Cavity Resonators
}

\author{
Marco Caiazzo \\ University of Siena \\ Stefano Maci \\ University of Siena \\ Nader Engheta \\ University of Pennsylvania, engheta@ee.upenn.edu
}

Follow this and additional works at: https://repository.upenn.edu/ese_papers

Part of the Electrical and Computer Engineering Commons

\section{Recommended Citation \\ Marco Caiazzo, Stefano Maci, and Nader Engheta, "A Metamaterial Surface for Compact Cavity Resonators", . January 2004.}

Copyright 2004 IEEE. Reprinted from IEEE Antennas and Wireless Propagation Letters, Volume 3, Issue 1, December 2004, pages 261-264. This material is posted here with permission of the IEEE. Such permission of the IEEE does not in any way imply IEEE endorsement of any of the University of Pennsylvania's products or services. Internal or personal use of this material is permitted. However, permission to reprint/republish this material for advertising or promotional purposes or for creating new collective works for resale or redistribution must be obtained from the IEEE by writing to pubs-permissions@ieee.org. By choosing to view this document, you agree to all provisions of the copyright laws protecting it.

This paper is posted at ScholarlyCommons. https://repository.upenn.edu/ese_papers/178

For more information, please contact repository@pobox.upenn.edu. 


\title{
A Metamaterial Surface for Compact Cavity Resonators
}

\begin{abstract}
We suggest an idea for miniaturization of cavities by utilizing a properly designed metamaterial thin surface inserted inside the cavities. This metamaterial surface is constituted by a thin dielectric slab on both sides of which "gangbuster" dipoles are printed. Inserting the thin slab inside a parallel-plate onedimensional (1-D) cavity resonator has the effect of decreasing the resonant frequency. Placing the metamaterial slab at the center of a rectangular waveguide also lowers the cut-off frequency of the dominant mode of the waveguide. The corresponding dispersion curve exhibits a smooth transition from a fast-wave to a slow-wave regime and then asymptotically tends to the dispersion curve of the first TE surface-wave mode of the metamaterial slab. This suggests a natural way to conceive a 3-D compact cavity resonator by placing two perfectly electric conducting walls, a half of the wavelength of the slowwave mode apart, inside the above rectangular waveguide. The analysis, performed by a circuit network theory and validated by a full-wave numerical analysis, provides simple formulas to predict the resonant frequency and the dispersion diagrams of these structures.
\end{abstract}

\section{Keywords}

Electromagnetic bandgap structures, frequency selective surfaces, metamaterials, periodic surfaces, resonators, transmission line networks, waveguides

Disciplines

Electrical and Computer Engineering

\section{Comments}

Copyright 2004 IEEE. Reprinted from IEEE Antennas and Wireless Propagation Letters, Volume 3, Issue 1, December 2004, pages 261-264. This material is posted here with permission of the IEEE. Such permission of the IEEE does not in any way imply IEEE endorsement of any of the University of Pennsylvania's products or services. Internal or personal use of this material is permitted. However, permission to reprint/republish this material for advertising or promotional purposes or for creating new collective works for resale or redistribution must be obtained from the IEEE by writing to pubspermissions@ieee.org. By choosing to view this document, you agree to all provisions of the copyright laws protecting it. 


\title{
A Metamaterial Surface for Compact Cavity Resonators
}

\author{
Marco Caiazzo, Student Member, IEEE, Stefano Maci, Fellow, IEEE, and Nader Engheta, Fellow, IEEE
}

\begin{abstract}
We suggest an idea for miniaturization of cavities by utilizing a properly designed metamaterial thin surface inserted inside the cavities. This metamaterial surface is constituted by a thin dielectric slab on both sides of which "gangbuster" dipoles are printed. Inserting the thin slab inside a parallel-plate one-dimensional (1-D) cavity resonator has the effect of decreasing the resonant frequency. Placing the metamaterial slab at the center of a rectangular waveguide also lowers the cut-off frequency of the dominant mode of the waveguide. The corresponding dispersion curve exhibits a smooth transition from a fast-wave to a slow-wave regime and then asymptotically tends to the dispersion curve of the first TE surface-wave mode of the metamaterial slab. This suggests a natural way to conceive a 3-D compact cavity resonator by placing two perfectly electric conducting walls, a half of the wavelength of the slow-wave mode apart, inside the above rectangular waveguide. The analysis, performed by a circuit network theory and validated by a full-wave numerical analysis, provides simple formulas to predict the resonant frequency and the dispersion diagrams of these structures.
\end{abstract}

Index Terms-Electromagnetic bandgap structures, frequency selective surfaces, metamaterials, periodic surfaces, resonators, transmission line networks, waveguides.

\section{INTRODUCTION}

$\mathbf{M}$ ETAMATERIAL media and surfaces with unconventional electromagnetic properties have attracted a great deal of attention and interest in recent years. Various ideas involving double-negative (DNG) media, single-negative (SNG) materials, electromagnetic band gap (EBG) structures, and artificial magnetic conductors (AMC) have been explored by many researchers over the past few years (see, e.g., [1], [2]). One such idea was the concept of compact, subwavelength waveguides and cavity resonators containing DNG or SNG materials, which were analyzed in detail in [3], [4]. Obviously, compactness of waveguides and cavities will offer advantages in design of miniaturized systems and subsystems. In the present letter, we explore a different approach to miniaturization of cavities and waveguides, namely, the use of frequency selective metamaterial surfaces inserted inside waveguides and cavities. As will be shown here, our numerical analysis demonstrates that such loading of waveguides and cavities with metamaterial surfaces may result in a significant reduction in cut-off and resonant frequencies, as compared with the corresponding unloaded elements.

Manuscript received April 14, 2004; revised July 15, 2004.

M. Caiazzo and S. Maci are with the Department of Information Engineering, University of Siena, Siena 53100, Italy (e-mail: macis@ing.unisi.it).

N. Engheta is with the Department of Electrical and Systems Engineering, University of Pennsylvania, Philadelphia, PA 19104 USA (e-mail: engheta@ee.upenn.edu).

Digital Object Identifier 10.1109/LAWP.2004.836576

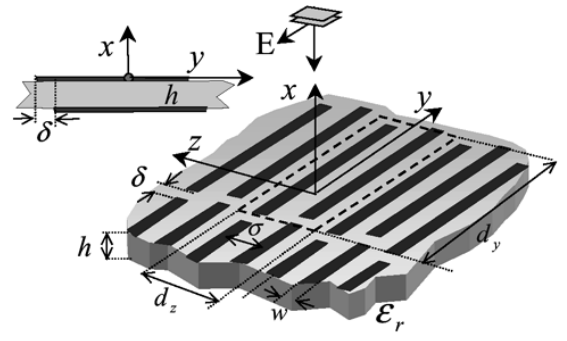

(a)

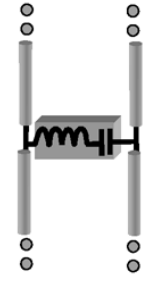

(b)
Fig. 1. Geometry of a gangbuster-FSS printed on a dielectric slab. $h=0.508$ $\mathrm{mm}, w=0.25 \mathrm{~mm}, d_{y}=10 \mathrm{~mm}, \delta=0.2 \mathrm{~mm}, d_{z}=0.5 \mathrm{~mm}$, relative permittivity of the slab $\varepsilon_{r}=4.5$. Dipoles are printed on both sides of the dielectric slab. Inset at the left: transverse cross section showing the offset of dipoles placed at the same z-level. (b) Equivalent $x$-transmission line circuit network.

\section{MEtAMATERIAL SLAB}

The basic geometry of the metamaterial thin slab we are dealing with is shown in Fig. 1. This surface consists of a frequency selective surfaces (FSS) formed by "gangbuster" dipoles [5] printed on both sides of a very thin dielectric slab. The dipoles are directed along the $y$ axis of a reference system with its $x$ axis along the normal to the surface, and its origin at the dielectric-air interface. The gangbuster surface is formed by the periodic repetition, with periods $d_{z}$ and $d_{y}$ along the $z$ and $y$ directions, of the basic periodic cell as shown by dashed lines in Fig. 1(a).

The reflection coefficient of the metamaterial slab for an incident plane wave with E-field polarized along the dipoles, can be described by the equivalent circuit network shown in Fig. 1(b) For the selected dimensions (with respect to the wavelength) all the Floquet waves (FWs) of the field expansion are evanescent along $x$, except for the dominant term. At a certain distance from the surface, where the evanescent FWs are significantly attenuated, we can assume that the field in the $y$ - $z$ plane is uniform in amplitude. Thus, the total field may be rigorously described by a transmission line along $x$ direction network with $x$-component of propagation constant and transverse impedance of the free space [5] [Fig. 1(b)]. The effect of the FSS (i.e., the metamaterial thin slab) is accounted for by a shunt impedance placed at $x=0$.

The extraction of the shunt reactance parameters from the infinite surface is performed by the pole-zero network synthesis suggested in [6]. For the geometrical parameters in Fig. 1(a), we have $C=2.404 \mathrm{pF}$ and $L=0.360 \mathrm{nH}$, corresponding to an equivalent impedance of the metamaterial slab as $Z_{\mathrm{FSS}}^{(0)}=$ $\left(1-\left(\omega / \omega_{0}\right)^{2}\right) / j \omega C$ where the resonant angular frequency of 


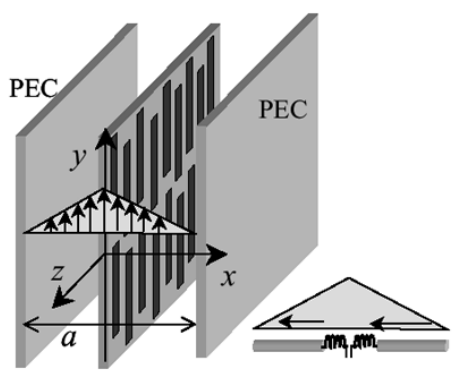

(a)

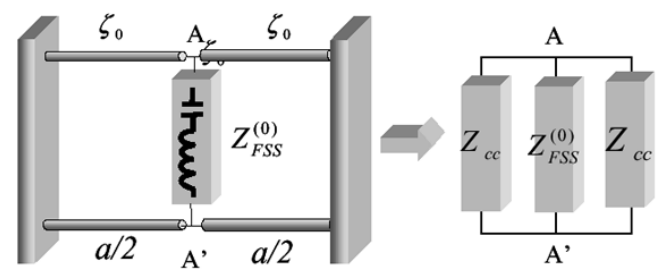

(b)

Fig. 2. (a) Geometry of 1-D cavity resonator $(a=15 \mathrm{~mm})$ and schematic profile of the dominant electric field along the $y$ axis. At the right hand side, an analogy with a short dipole loaded with lumped inductances at the delta gap is shown. (b) Equivalent $x$-transmission line network (left-hand side) and its corresponding impedances as observed at the midpoint section (right-hand side).

FSS is $\omega_{0}=(1 / \sqrt{L C})=2 \pi \times 5.41 \mathrm{GHz}$. The $L$ and $C$ parameters in general depend on the permittivity of the dielectric slab; however since the slab is assumed to be very thin, its permittivity does not play a major role in our analysis here.

\section{1-D CAVITY RESONATOR}

The metamaterial slab described in the previous section is now placed at the midpoint between two perfectly electric conducting (PEC), infinitely extent parallel walls separated by a distance $a$ [Fig. 2(a)]. To illustrate the idea, we consider $a=15$ $\mathrm{mm}$. The structure realizes a one-dimensional (1-D) resonator which can be modeled as an equivalent transmission line with characteristic impedance of free-space [Fig. 2(b)]. We are interested in lowering the dominant resonant frequency of this 1-D cavity resonator, which has its electric field along the $y$ axis, by inserting the metamaterial thin slab.

Intuitively, we expect to achieve a lower resonant frequency compared with that of the unloaded parallel-plate waveguide. Indeed, at a low frequency the metamaterial slab behaves essentially as a capacitive reactance, and this provides reduction and cancellation of the inductive reactance provided by the two walls as observed from the mid point. Thus, we expect that the $E_{y}$ modal field distribution possesses an average triangular profile, in analogy with a thin resonant electric dipole fed at the delta gap by a lumped inductance [this latter exhibits a triangular profile of electric currents, as shown in the inset of Fig. 2(a)].

In order to find the resonant frequency of the loaded 1-D cavity resonator, we consider the equivalent $x$-transmission line circuit network in Fig. 2(b). The metamaterial slab is represented with a shunt L-C series circuit at the center [Fig. 2(b)]. The inductance and the capacitance of this shunt element can be designed using the corresponding infinite metamaterial slab in Fig. 1(a). Insertion of these parameters in the equivalent network in Fig. 2(b) will be justified, if we assume that the interaction between the metamaterial slab and the side walls occurs through only the dominant Floquet wave of the FSS-slab field expansion. This assumption is reasonable since the surface can be treated as being effectively "homogeneous" along the $z$ direction. In the next section, we will check this assumption a posteriori by comparing it with a more rigorous full-wave analysis.

On the basis of the transmission-line circuit network in Fig. 1(b), the transverse resonance equation is obtained by imposing the $2 / Z_{c c}+1 / Z_{\mathrm{FSS}}=0$, where $Z_{c c}=j \xi_{0} \tan \left((1 / 2) a k_{0}\right)$ is the short circuit impedance as observed at the center of the structure, and $Z_{\mathrm{FSS}}$ is the shunt impedance representing the FSS-slab element. This leads to the following equation for the resonant frequency $\omega_{\text {res }}$

$$
\frac{\omega_{\mathrm{res}} C}{1-\left(\omega_{\mathrm{res}} / \omega_{0}\right)^{2}}=\frac{2}{\xi_{0} \tan \left(\frac{1}{2 c} a \omega_{\mathrm{res}}\right)}
$$

where $c$ is the free-space speed of light (since we assume the region between the walls and the slab is free space). Using the small-argument approximation for the tangent function, we obtain the following closed-form expression

$$
\omega_{\mathrm{res}} \approx 1 / \sqrt{\frac{1}{4} C \mu_{0} a+1 / \omega_{0}^{2}} .
$$

For the dimensions shown in Fig. 1(a), the resonant frequency from (2) is found to be $f_{\text {res }}=\omega_{\text {res }} 2 \pi=1.44 \mathrm{GHz}$ (while (1) yields $1.43 \mathrm{GHz}$ ); for this value of frequency, the argument of the tangent is very small, thus justifying the approximation used to obtain (2). We note that the unloaded parallel plate empty waveguide resonates at $f_{\text {res }}^{\prime}=\mathrm{c} / 2 a=10 \mathrm{GHz}$. Thus, the slab loading with metamaterial-FSS slab has lowered the resonance frequency by a factor of

$$
\omega_{\mathrm{res}}^{\prime} / \omega_{\mathrm{res}} \approx \frac{\pi}{2} \sqrt{C /\left(a \varepsilon_{0}\right)} \approx 6.9
$$

It is worth noting that if we had loaded the 1-D cavity with the fin line or the ridge with the same thickness and same gap size as the one in the gangbuster FSS here, according to our numerical analysis the resonant frequency would have reduced by a factor of 3.35 and 2.58 , respectively, which is smaller than what we have in (3).

\section{Metamaterial-Thin-Slab LOADED WAVEGUIDE}

Let us now consider the metamaterial slab placed in the center of a rectangular waveguide with dimensions $a \times b$, in parallel with its two side walls (Fig. 3). For example we assume $a=15$ $\mathrm{mm}$ (as in the case of 1-D cavity resonator) and $b=5 \mathrm{~mm}$, i.e., with $b=d_{y} / 2$. The transverse cross section of the waveguide obviously represents a 2-D rectangular resonator, possessing the first resonant frequency as the first cut-off frequency of the rectangular waveguide.

In its dominant mode, the structure may be regarded as the 1-D resonator with an additional pair of walls orthogonal to the $y$ axis. Since the dipoles are very thin and are tightly packed, the electric currents on the dipoles are directed along the $y$ axis and the electric field has a dominant $y$-component; thus, effectively removing the upper and lower PEC walls, yields the geometry 


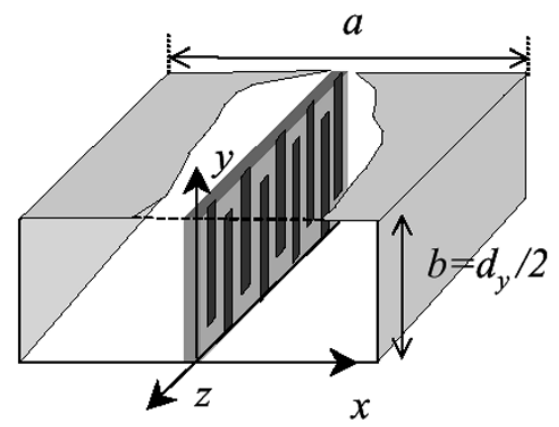

(a)

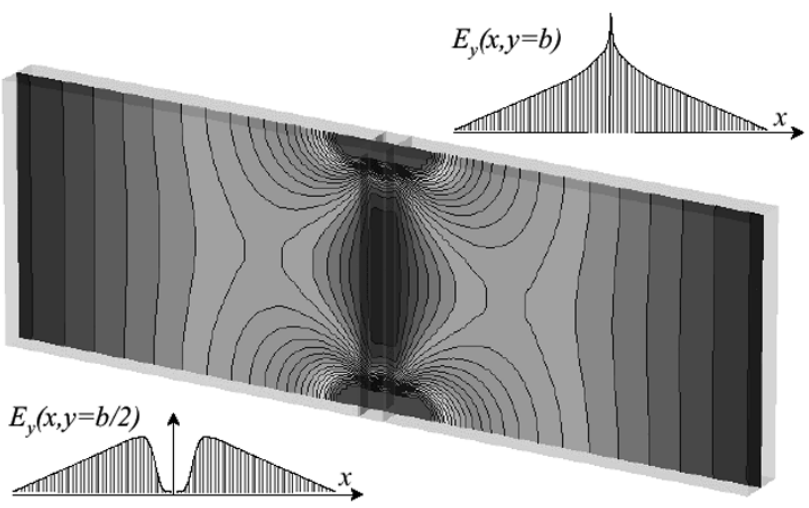

(b)

Fig. 3. (a) Geometry of the waveguide loaded with the metamaterial slab ( $a=$ $15 \mathrm{~mm}, b=5 \mathrm{~mm}$ ). (b) Equimagnitude lines of the $E y$ field at the resonant frequency $f=1.41 \mathrm{GHz}$.

of the 1-D resonator. Therefore, we expect that the model in Fig. 2(b) and the corresponding result in (1) also applies to this waveguide dominant cut-off frequency.

To verify, the structure has also been analyzed by using the commercial software code CST Microwave Studio. The periodicity condition with a zero phase shift has been set for two walls orthogonal to the $z$ direction at a distance $d_{z}$. A resonant mode has been found at $f_{\text {res }}=1.41 \mathrm{GHz}$, which agrees well with the one predicted by the 1-D theoretical model, i.e., $1.43 \mathrm{GHz}$, from (1). Fig. 3(b) shows the level contour lines of $E_{y}$ for the resonant mode for the 2-D cavity resonator in Fig. 3(a). The reactive field is concentrated near the interdipole gaps, and decreases to zero at the PEC walls with a quasi-linear profile. In the two insets, the schematic profile of $E_{y}$ versus $x$ is depicted for two sections at the center $(y=b / 2)$ and close to the upper wall at $(y=b)$.

As mentioned earlier, the first resonant frequency associated with the 2-D cavity resonator, provided by (1), approximates the dominant cut-off frequency of the waveguide. Increasing the frequency beyond this resonance leads to a modal propagation with a $k_{z}$ wavenumber along the waveguide. The dispersion equation $k_{z}=k_{z}(\omega)$ can be obtained, starting from the TE equivalent network model in Fig. 4.

The equivalent impedance $Z_{\mathrm{FSS}}^{\mathrm{TE}}\left(k_{z}, \omega\right)$ representing the metamaterial slab has been determined by the pole-zero network synthesis presented in [6]. An oblique incident TE plane wave can then be considered for the problem in Fig. 1.

The equivalent impedance of FSS is weakly dependent on $k_{z}$, due to the weak dependence of the FSS resonance on the direc-

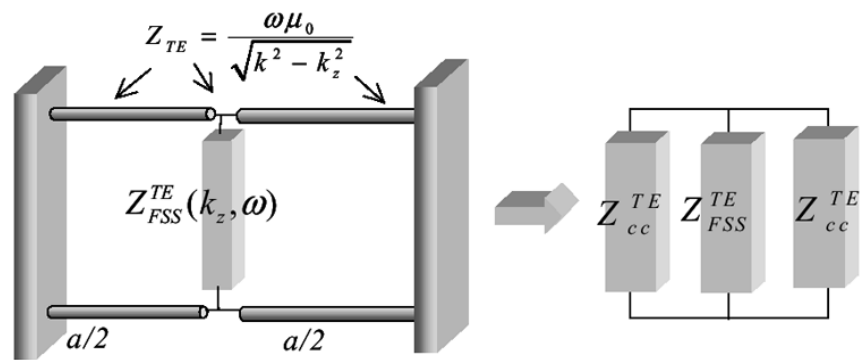

Fig. 4. Equivalent dispersive $x$-transmission line circuit network (left) and corresponding impedances as observed at the mid point section (right).

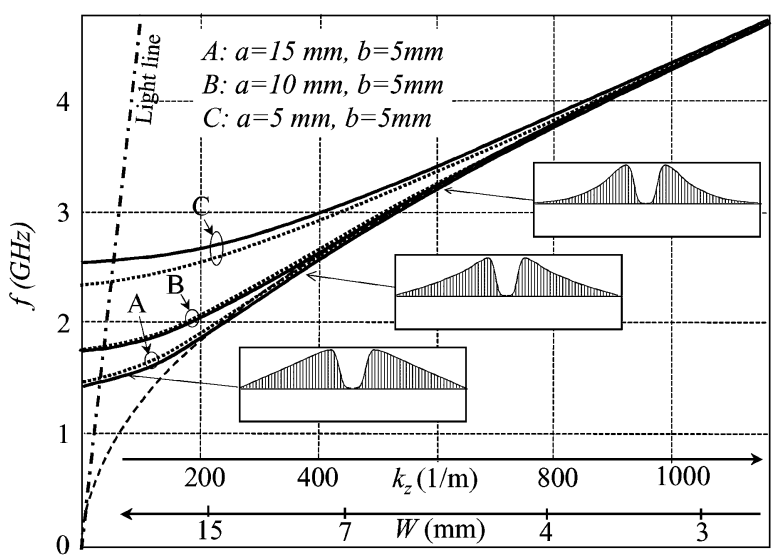

Fig. 5. Dispersion curves $\left(f-k_{z}\right)$ of the metamaterial-slab-loaded waveguide for different size a. Continuous line and dotted lines refer to result from CST and from (5), respectively. Dashed line is relevant to the dispersion curve of the first surface wave TE mode of the metamaterial thin slab. The horizontal axis exhibits a second scale, which serves to find the resonant frequency of a 3-D cavity with length $\mathrm{W}$. The cross-sectional field distribution insets show how the fields decreases for different point along the dispersion curve. As one gets farther away from the light line, the field decays more rapidly.

tion of the incident wave. However, this dependence has been found to be important for a good predictive model. A single pole impedance is still used to describe the frequency dependence of the equivalent impedance; i.e., $Z_{\mathrm{FSS}}^{\mathrm{TE}}\left(k_{z}, \omega\right)=j \omega C /[1-$ $\left.\omega^{2} / \omega_{p}^{2}\right]$. However, the pole is found to be linearly dependent on $k_{z}$ as $\omega_{p}\left(k_{z}\right)=\omega_{0}+\eta c k_{z}$, where $c$ is the speed of light in free space and $\omega_{0}$ is the resonant angular frequency of the FSS found for normal incidence. The dimensionless constant $\eta=5,5 \times 10^{-3}$ has been determined by matching (in the visible range $k_{z}<k$ ) the full-wave data with the response from the transmission line network in Fig. 4. Analytical continuation of $\omega_{p}\left(k_{z}\right)$ results in the analytical form $2 / Z_{c c}^{\mathrm{TE}}+1 / Z_{\mathrm{FSS}}^{\mathrm{TE}}=0$ of the transverse resonance equation, where $Z_{c c}^{\mathrm{TE}}$ is the short-circuit impedance as observed at the center of the structure; this yields

$$
\frac{2 \sqrt{k^{2}-k_{z}^{2}}}{j \omega \mu_{0} \tan \left(\frac{1}{2} a \sqrt{k^{2}-k_{z}^{2}}\right)}+\frac{j \omega C}{1-\left(\omega /\left(\omega_{0}+\eta c k_{z}\right)\right)^{2}}=0 .
$$

The dispersion curves are presented in Fig. 5 for various values of the dimension $a$. These curves are compared with those obtained by CST Microwave Studio ${ }^{\mathrm{TM}}$, used by imposing a periodicity condition with a phase shift of $d_{z} k_{z}$. The full-wave dispersion curves agree well with those provided by (4), except when $a$ is very small and the higher-order FWs associated with the 


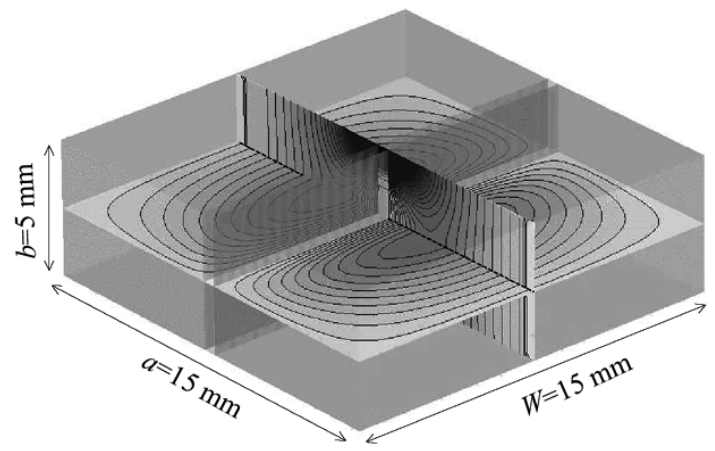

Fig. 6. Geometry of a 3-D resonator, and modal $E y$-field distributions in three cross-sectional planes, at the frequency of $1.866 \mathrm{GHz}$. Dimensions: $a \times b \times W=$ $15 \times 5 \times 15 \mathrm{~mm}$

metamaterial FSS slab interact significantly with the side walls. Starting from a cut-off frequency, as the frequency increases, the dispersion curves crosses the light-line (dashed line), and then moves toward the slow-wave region. Beyond the light line, the dominant FW associated with the metamaterial thin slab is evanescent, along $x$. The dispersion equation (4) may be conveniently rewritten as

$$
\frac{2 \alpha}{\omega \mu_{0} \tanh \left(\frac{1}{2} \alpha a\right)}=\frac{\omega C}{1-\left(\omega /\left(\omega_{0}+\eta c k_{z}\right)\right)^{2}}
$$

where $\alpha=\sqrt{k_{z}^{2}-k^{2}}$ is the attenuation constant of the dominant FW along $x$. As the frequency increases further, all the curves asymptotically blend into the dispersion curve of the metamaterial FSS slab surrounded by free space. This asymptotic behavior can be mathematically obtained by setting $a \alpha \gg$ 1 in (5) resulting in the hyperbolic tangent being unity. This implies vanishing of the dominant FW field at the side walls.

\section{3-D CAVITY RESONATOR}

A corresponding 3-D compact resonator can be obtained by inserting two additional PEC walls a distance $W$ apart and orthogonal to the $z$-direction inside the loaded waveguide (Fig. 6). The first resonance of this 3-D cavity resonator is found by imposing $\pi / W=k_{z}$, that is by assuming the cavity length being one half of the slow-wave mode's wavelength. From (5), this leads to

$$
\begin{aligned}
& \frac{2 \sqrt{\left(\frac{\pi}{W}\right)^{2}-\left(\frac{\omega_{\text {res }}}{c}\right)^{2}}}{\omega_{\text {res }} \mu_{0} \tanh \left(\frac{1}{2} \alpha \sqrt{\left(\frac{\pi}{W}\right)^{2}-\left(\frac{\omega_{\text {res }}}{c}\right)^{2}}\right)} \\
& =\frac{\omega_{\text {res }} C}{1-\left(\omega_{\text {res }} /\left(\omega_{0}+\eta c \pi / W\right)\right)^{2}}
\end{aligned}
$$

where $\omega_{\text {res }}$ is the resonant frequency of this 3-D resonator. (The graphical interpretation may be deduced from Fig. 5 reading the lower horizontal scale). When $\omega_{\text {res }} \ll c \pi / W$, (6) may be approximated as

$$
\begin{aligned}
\omega_{\mathrm{res}}=\left[\frac{1}{2 \pi} W C \mu_{0} \tanh \left(\frac{\pi}{2} \alpha / W\right)\right. & \\
& \left.+\left(\omega_{0}+\eta c \pi / W\right)^{-2}\right]^{-1 / 2}
\end{aligned}
$$

It is interesting to compare this resonant frequency with that associated with the $\mathrm{TE}_{101}$ mode in the empty rectangular cavity resonator

$$
\omega_{\mathrm{res}}^{\prime}=\pi c\left[(1 / W)^{2}+(1 / a)^{2}\right]^{1 / 2} .
$$

For an illustrative example, consider the case $W=a=15 \mathrm{~mm}$ (Fig. 6). The resonant frequency predicted by (7) is $f_{\text {res }}=1.93$ $\mathrm{GHz}$, while for the empty cavity we have, from (8), $f_{\text {res }}^{\prime}=14.14$ $\mathrm{GHz}$; thus, obtaining a "compression ratio" of $f_{\text {res }}^{\prime} / f_{\text {res }}=7.32$. Using CST, a resonance is found at $f_{\text {res }}=1.87 \mathrm{GHz}$, which agrees well with that predicted by (7). If instead, we had used the fin line or the ridge, the compression ratio would have been 2.06 and 1.79 , respectively. The field distributions in the $x-y$ plane $(z=W / 2)$ and in the $x-z$ plane $(y=b / 2)$ are shown in Fig. 6.

\section{CONCLUDING REMARK}

In this letter, it has been shown theoretically how a metamaterial surface (i.e., thin FSS slab) simply composed of gangbuster dipoles printed on both sides of a thin dielectric slab can be used to significantly lower the resonant frequency of a cavity resonator. The parallel plate (1-D) resonator exhibits approximately a lowering of the resonant frequency by about a factor of $(\pi / 2) \sqrt{C /\left(a \varepsilon_{0}\right)}$, where $C$ is the equivalent capacitance of the metamaterial surface. For the corresponding 3-D cavity resonators, further reduction of the resonant frequency can be achieved by loading such metamaterial surfaces. This technique can provide new methods for the design of compact, ultrathin cavity resonators without using high-permittivity dielectric fillers.

\section{REFERENCES}

[1] R. Ziolkowski and N. Engheta, Eds., IEEE Trans. Antennas Propagat., Oct. 2003, vol. 51. Special issue on Metamaterials.

[2] J. Pendry, Ed., Negative Refraction and Metamaterials, 2003, vol. 11. Focus Issue of Optics Express on.

[3] N. Engheta, "An idea for thin subwavelenght cavity resonator using metamaterial with negative permittivity and permeability," IEEE Antennas Wireless Propagat. Lett., vol. 1, pp. 10-13, 2002.

[4] A. Alu and N. Engheta, "Guided modes in a waveguide filled with a pair of single-negative (SNG), double-negative (DNG), and/or doublepositive (DPS) layers," IEEE Trans. Microwave Theory Tech., vol. 52, pp. 199-210, Jan. 2004.

[5] B. A. Munk, Frequency Selective Surfaces: Theory and Design. New York: Wiley, 2000.

[6] S. Maci, M. Caiazzo, A. Cucini, and M. Casaletti, "A network theory of EBG surfaces," IEEE Trans. Antennas Propagat., to be published. 